\title{
Imaging with Fresnel zone pupil masks: extended depth of field
}

\author{
Guy Indebetouw and Hanxian Bai
}

\begin{abstract}
Fresnel zone plates are used as pupil apertures to extend the depth of field of an incoherent imaging system. The defocused optical transfer function is evaluated approximately, and the results are confirmed by experiments and by subjective evaluations of the images of a test chart. With a pupil having four zones, for example, the resolution limit at four depths of field out of focus is improved by a factor of 10 .
\end{abstract}

\section{Introduction}

The information gathered by an image forming instrument is essentially limited by the size of its pupil. With a clear circular pupil of numerical aperture $\alpha$, and a radiation of wavelength $\lambda$, the lateral and longitudinal resolutions in the image space are $\lambda / 2 \alpha$ and $\lambda / \alpha^{2}$, respectively. Although these are basic limitations, it is nevertheless possible to change the imaging properties of the system by using different kinds of pupil mask. For example, it is often desirable, in applications where the information is gathered in a plane, to have a larger depth of field than that given by the longitudinal resolution. Thin annular apertures have been used for this purpose. ${ }^{1}$ The defocused optical transfer function (OTF) with an annular pupil is largely independent of the amount of defocusing and remains real positive for all spatial frequencies. It, however, has a very small magnitude except for very low spatial frequencies. The low intensity and contrast of the resulting images may be unacceptable if noise is present in the system.

The purpose of this paper is to investigate the properties of an incoherent imaging system using Fresnel zone plates (FZP) as pupil masks. The large depth of field of a thin annular aperture is due to the fact that all the aperture elements contribute inphase at any axial position in the image space. With a FZP aperture, each zone behaves as an annulus, but the contributions from different zones are inphase only at these axial positions corresponding to the foci of the combined lens-FZP system. The expected gain in depth of field is, therefore, smaller than that of an annular aperture, but the image intensity and contrast will be greatly improved.

The authors are with Virginia Polytechnic Institute and State University, Physics Department, Blacksburg, Virginia 24061.

Received 28 June 1984.

0003-6935/84/234299-04\$02.00/0.

(C) 1984 Optical Society of America.
An approximate expression for the defocused OTF is derived and discussed in the next section. It is followed by some illustrations and the results of a comparative evaluation of images obtained with zone plates of various powers.

\section{Defocused OTF}

The generalized pupil function ${ }^{2}$ of a centrosymmetric optical system having a FZP pupil can be written as

$$
P(\rho, z)=\sum_{n} A_{n} \exp \left[i 4 \pi(z+n N) \rho^{2}\right] \operatorname{circ} \rho,
$$

where the radial spatial frequency $\rho$ is normalized to the cutoff frequency $W / 2$ of the clear pupil, the lateral coordinates in the image plane are measured in units of resolution limit $W^{-1}$, and the axial coordinate $z$ is measured in units of depth of field $4 / \lambda W^{2}$. The FZP used in Eq. (1) has an amplitude transmittance

$$
T(\rho)=\sum_{n} A_{n} \exp \left(i 4 \pi n N \rho^{2}\right),
$$

where $N$ is the number of zones in the pupil (half of them opaque). The various spherical waves diffracted by the FZP come to focus at axial distances of $N$ depths of field from each other. For opaque and transparent zones of equal area, $A_{n}=( \pm 1)^{n} \sin (n \pi / 2) / n \pi$, where the upper/lower sign corresponds to a clear/opaque center zone, respectively.

An approximate expression for the defocused OTF can be obtained in the following manner. Changing to Cartesian coordinates, a trace along the axis $v=0$ through the OTF can be written as the Fourier transform of a projection along $u^{\prime}$ of two superposed pupil apertures shifted by $u$ :

$$
H(u, o, z)=\int \phi\left(u^{\prime}, u\right) \exp \left(-i 8 \pi z u^{\prime} u\right) d u^{\prime},
$$

where

$$
\phi\left(u^{\prime}, u\right)=\int P\left(u^{\prime}+u / 2, v^{\prime}\right) P^{*}\left(u^{\prime}-u / 2, v^{\prime}\right) d v^{\prime},
$$

and normalization factors are omitted. Using Eq. (1) and assuming for simplicity that the pupil $P$ is bounded by a square of side 1 , the product of the two shifted apertures becomes 

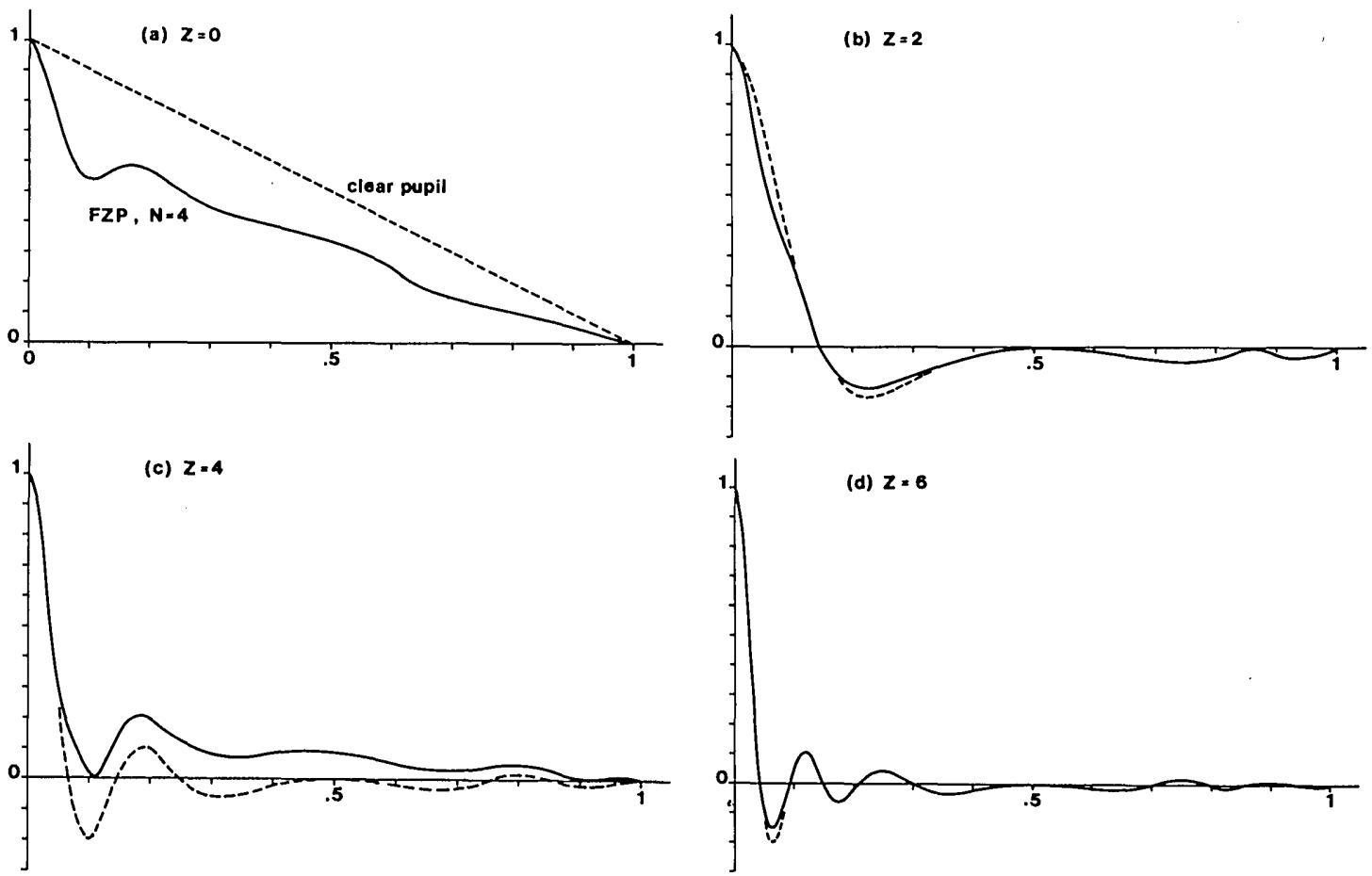

Fig. 1. Approximate defocused OTF for an FZP pupil having four zones compared with the OTF of a clear aperture of the same size (a) in focus $(z=0)$; (b) two depth of field out of focus $(z=2)$; (c) $z=4$; (d) $(z=6)$.

$$
\begin{aligned}
P(+) P^{*}(-)= & \sum_{m, n} A_{m} A_{n} \exp \left\{i 4 \pi N \left[(m-n)\left(u^{\prime 2}+v^{\prime 2}+u^{2} / 4\right)\right.\right. \\
& \left.\left.+(m+n) u^{\prime} u\right]\right\} \operatorname{rect}\left(v^{\prime}\right) \operatorname{rect}\left[u^{\prime} /(1-u)\right] .
\end{aligned}
$$

For an aperture having a sufficiently large number of zones $(n>4)$, the phase term in $v^{2}$ oscillates rapidly enough to contribute to the integral of Eq. (4) only if $m$ $=n$ (stationary phase point). The OTF then takes the much simpler form

$$
H(u, o, z) \sim \Sigma A_{n}^{2} \delta(z-n N) * \sin [4 \pi z u(1-u)] /(4 \pi z u) .
$$

The interpretation of this result is simple. In constructing the projections $\phi$, all the moire features of the two superposed zone plates average out except for the Schuster fringes, ${ }^{3}$ which contribute to a modulation of $\phi$ having a spatial frequency $8 \mathrm{Nu}$. For certain amounts of defocusing, these modulations result in large values of the OTF. The consequence of neglecting all the cross terms in Eq. (5) in that the defocused OTF is simply the sum of contributions from clear apertures defocused by amounts $z-n N$. In the image space, the PSF is, therefore, the sum of the corresponding PSF weighed by $A_{n}^{2}$ and axially spaced by $N$. Neglecting the interferences between these contributions is reasonable if $N$ $>4$ or 5 , which is compatible with the initial assumption used in deriving Eq. (6). More exact calculations taking these interactions into account can be made numerically. ${ }^{4}$ It is sufficient to note here that for $n<3$, the aperture is simply a stopped down pupil or an annulus, cases which have been studied in the literature. ${ }^{1,5}$

Figure 1 shows some defocused OTF with a pupil aperture having $N=4$ zones. Only the three first orders of the approximate expression (6) were kept. In

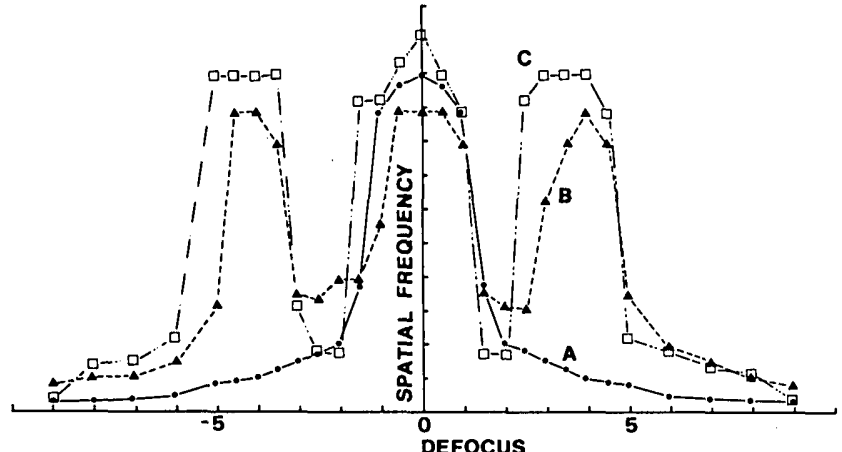

(a)

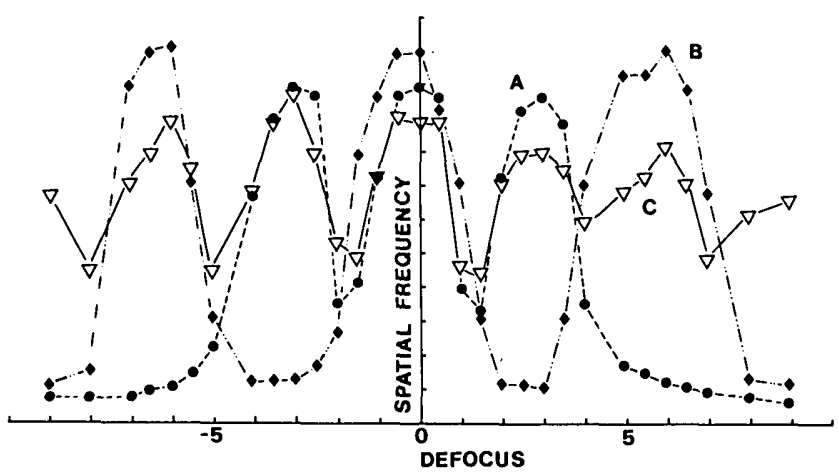

(b)

Fig. 2. Evaluation of the quality of defocused images obtained with FZP apertures having different numbers $N$ of zones. The measured parameter is the spatial frequency of a test chart corresponding to the limit of visual detection: (a) $A, N=0$ (clear circular aperture), $B, N$ $=4^{(+)}$(four zones, clear center), $C, N=4^{(-)}$(four zones, dark center); (b) $A, N=3^{(+)}$(three zones, clear center), $B, N=6^{(-)}$(six zones, dark center), $C$, superposition of three zones, clear center and six zones, dark center. 
A

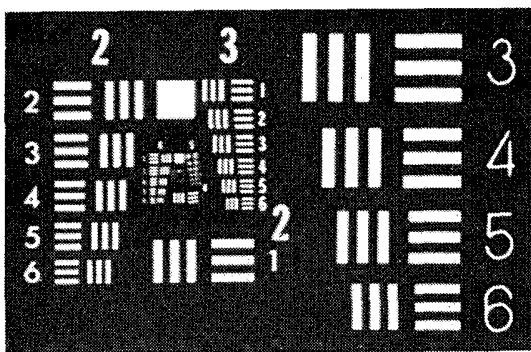

a

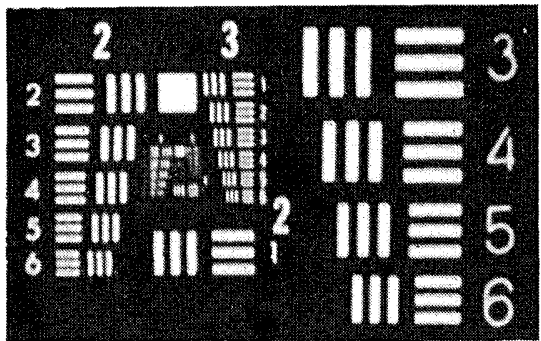

b

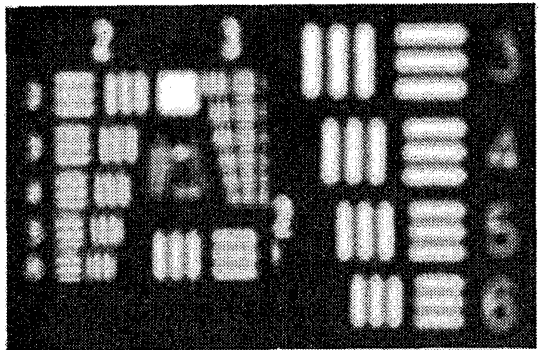

C

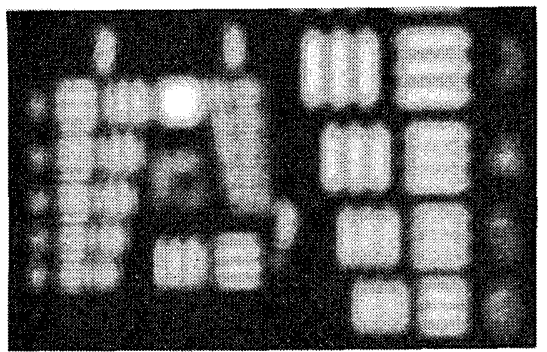

B

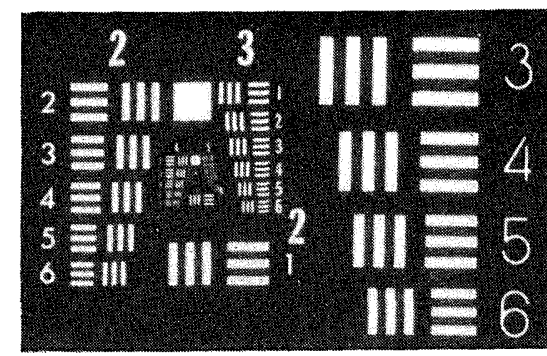

a

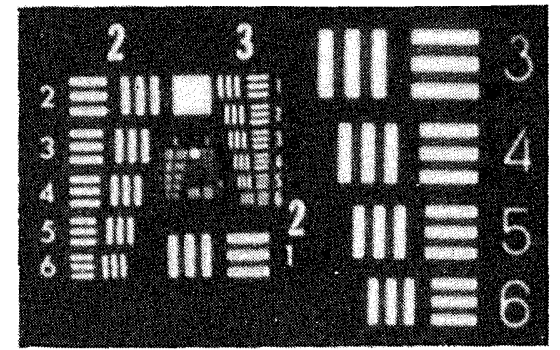

b

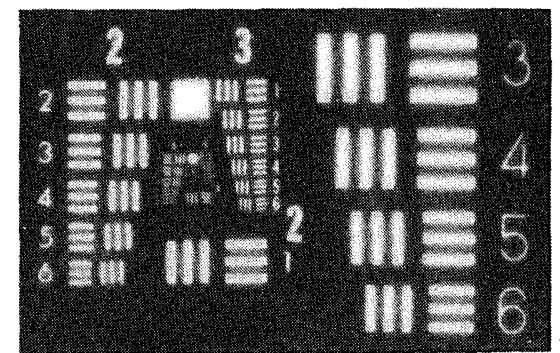

C

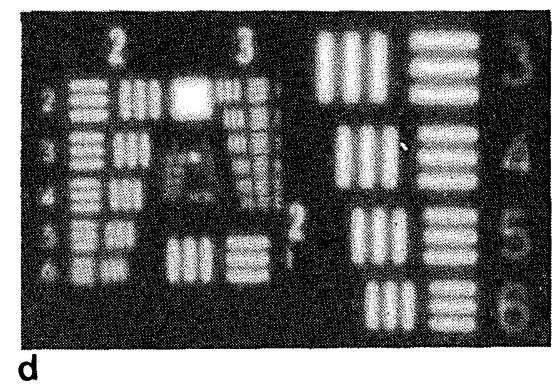

C

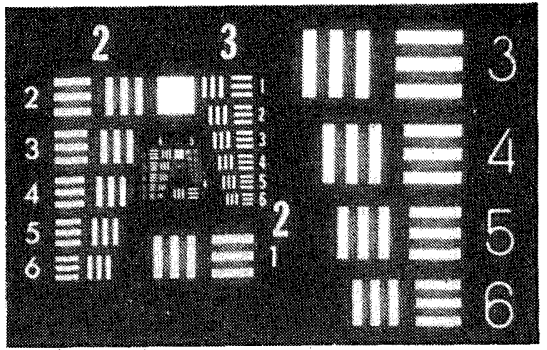

a

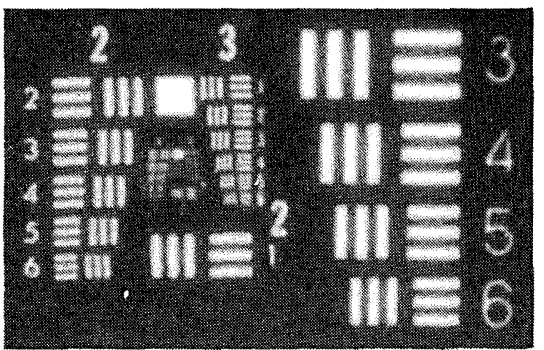

b

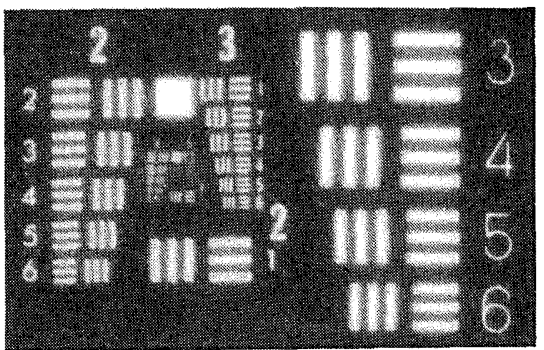

C

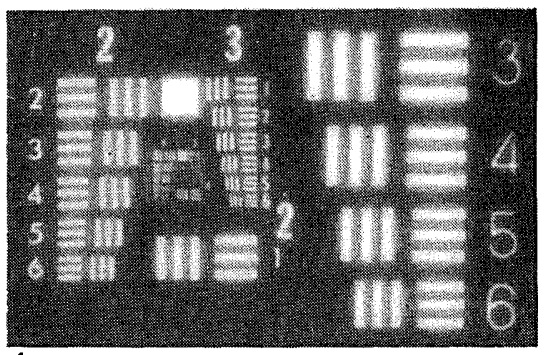

d

Fig. 3 (A) Clear circular aperture $(N=0)$ (a) in focus, $z=0$, (b) two depth of field defocus, $z=2$, (c) $z=4$, (d) $z=6$. (B) Pupil with four zones, clear center $(N=4)$, (a)-(d) as in (A). (C) Pupil with six zones, clear center $(N=6)$; (a)-(d) as in (A).

focus [Fig. 1(a)], the degradation of the OTF is not excessive as it would be, for example, with an annular pupil. A very substantial gain is obtained at $z=4$, where the first order of the FZP comes to focus [Fig. $1(\mathrm{c})]$. At this position, the OTF has no contrast inversion and is much larger in magnitude than the corresponding $\mathrm{OTF}$ of a clear pupil or a thin annulus. This gain extends for an axial range of $\sim 2$ depths of field. At $z<2$ and $z>6$, the OTF is essentially the same as that of a clear pupil of the same size.

\section{Experimental Results}

Quantitative data for the quality of an image obtained with various amounts of defocusing and with several pupil apertures were gathered by measuring the spatial frequency limit of visual detectability in the image of a test chart formed by an afocal system. The measure is subjective but, if consistent, nevertheless a good indication of the image quality including factors of resolution and contrast. Some of the results are summarized in Figs. 2(a) and (b). Figure 2(a) confirms the previous analysis and evaluation of the gain of depth of field. In that picture, $N^{(+)}$and $N^{(-)}$refer to apertures with a clear or an opaque central zone, respectively. The asymmetry between the result obtained with complementary zones, for small $N$, is of course not predicted by the previous approximate theory. In Fig. $2(\mathrm{~b})$, a superposition of two FZP $\left[N=6^{(-)}\right.$and $\left.N=3^{(+)}\right]$ 


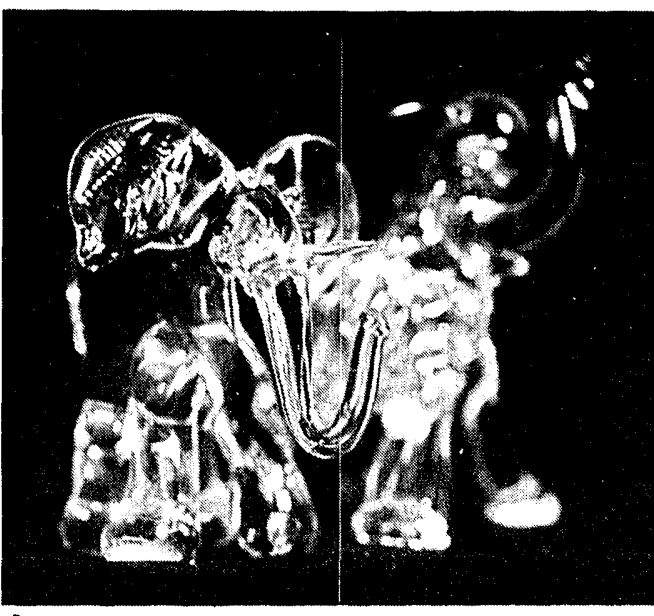

A

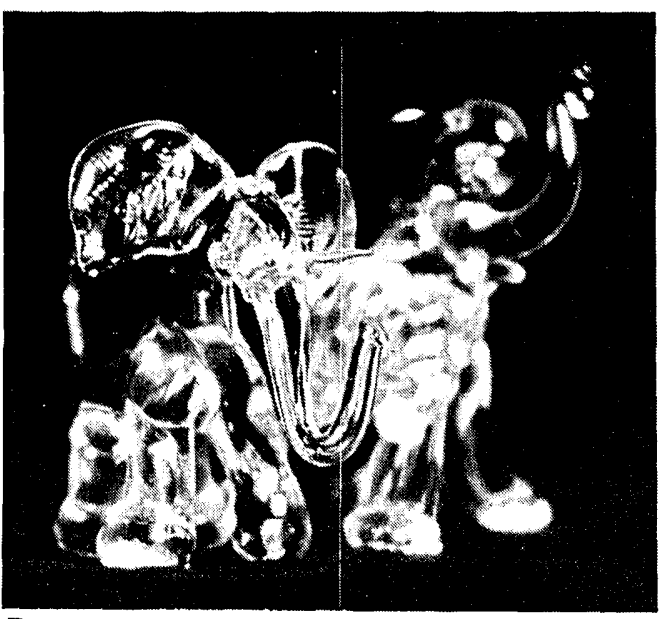

B

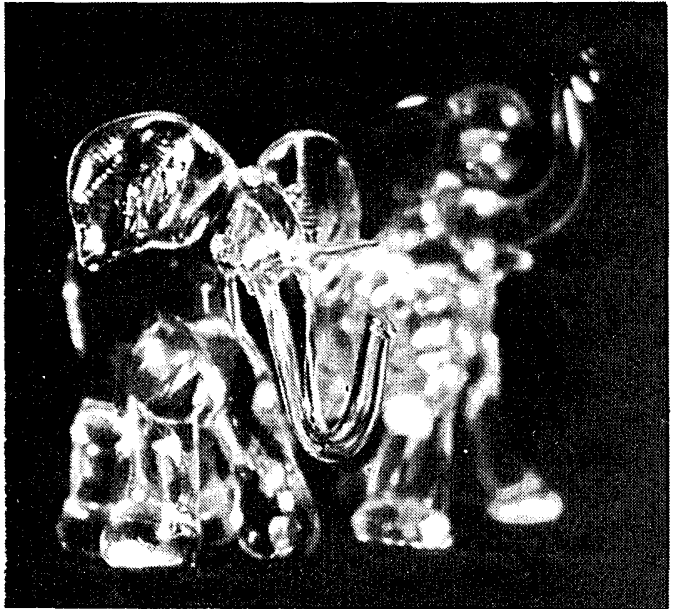

C

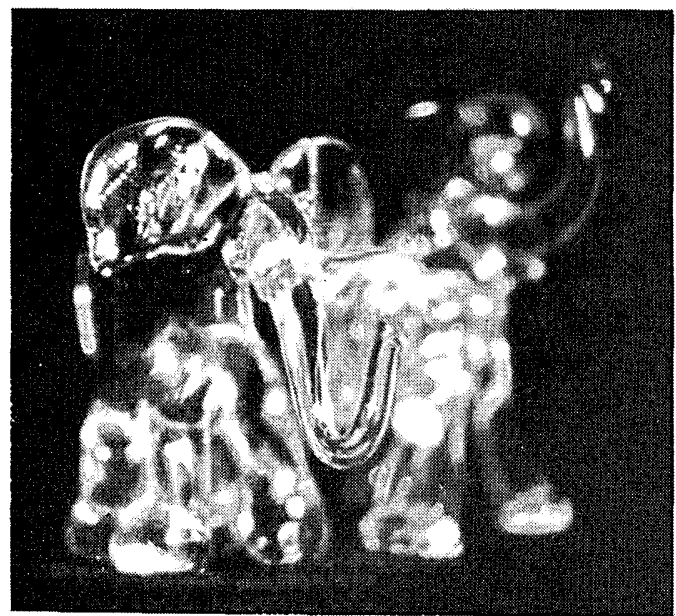

D

Fig. 4. (A) Clear circular aperture; (B) FZP pupil with five zones, clear center; (C) superposition of FZP $N=3^{(+)}$and $6^{(-)}$; (D) thin annular aperture of $\sim 0.1$ width.

was also used as an aperture. As an approximate theory neglecting all cross terms would predict, the result is close to a simple superposition of the result of each aperture taken separately. This results in an additional gain in depth of field together with a reduction of the transparent pupil area and, therefore, of image intensity. Some images of test charts obtained with different apertures and various amounts of defocusing are shown in Fig. 3. Figure 4 shows pictures of a figurine taken with a clear circular aperture (A) with a FZP pupil having five zones (B). For comparison, Fig. 4(C) shows a picture taken with a pupil made of a superposition of two FZP $\left[N=3^{(+)}\right.$and $\left.N=6^{(-)}\right]$, while Fig. $4(D)$ was obtained with a thin annular pupil (width $\sim 0.1$ ).

\section{Conclusions}

Fresnel zone plates can be used in the pupil of an incoherent imaging system to extend its depth of field without reducing excessively image intensity and contrast. The image properties can be analyzed by an approximate but simple expression of the defocused OTF, which results from neglecting the cross terms in the superposition of pupil terms with various quadratic phases. The results are confirmed by some experiments.

The financial support of the National Science Foundation (grant ECS 8116337) for the research leading to this work is gratefully acknowledged.

\section{References}

1. W. T. Welford, "Use of Annular Apertures to Increase Focal Depth," J. Opt. Soc. Am. 50, 749 (1960); J. T. McCrickerd, “Coherent Processing and Depth of Focus of Annular Aperture Imagery," Appl. Opt. 10, 2226 (1971).

2. H. H. Hopkin, Wave Theory of Aberrations (Oxford U. P., London, 1950).

3. A. W. Lohman, Optical Information Processing (Physikalisches Institut der Universitat, 8520 Erlangen, EDR, 1978).

4. C. J. C. Thompson and W. L. Wolfe, "Three-Dimensional Intensity Distribution in the Region of Focus of Two Superposed Converging Spherical Waves," J. Opt. Soc. Am. A 1, 356 (1984).

5. M. Mino and Y. Okano, "Improvement in the OTF of a Defocused Optical System Through the Use of Shaded Apertures," Appl. Opt. 10, 2219 (1971). 